\title{
On the Time of Amber Light at Crossroads
}

\author{
Meng Xinyu, a, Zhao Jian", b, Zhang Wei ${ }^{3, c}$, Meng Zhaoping ${ }^{4, d}$ \\ ${ }^{1}$ School of Automation and Electronic Engineering, Qingdao University of Science and Technology, \\ Qingdao, P.R. China \\ ${ }^{2}$ School of Environment and Safety Engineering Qingdao University of Science and Technology \\ Qingdao, P.R. China \\ ${ }^{3}$ School of Information Science and Technology, Qingdao University of Science and Technology, \\ Qingdao, P.R. China \\ ${ }^{4}$ School of Information Engineering, Shandong Youth University of Political Science, Jinan, P.R. \\ China \\ a995273735@QQ.com, ${ }^{b} 715171198 @ Q Q . c o m,{ }^{c} 460825550 @ Q Q . c o m,{ }^{d}$ Mengzhp@yeah.net
}

Keywords: difficult area; sensitivity analysis; differential equation; image rendering

Abstract: According to the relationship between the speed of vehicle and the amber light, the differential equation model of the amber light duration is established. And based on the relevant conditions given in the title, three differential equation models of amber light duration under different conditions are obtained. Considering the traffic condition and driver's habit, a value that is most suitable for the actual demand is calculated. The sensitivity and stability of the model and its related factors are analyzed. The model for the problem of difficult area is improved.

\section{Introduction}

When you are in the traffic lights, are you noticed how long the amber light is on? In fact, the setting of the amber light duration has many limitations and plays a crucial role. So, have you ever thought about how long the amber light should be on? What are the restrictions on the length of the amber light? In traffic management, amber lights are set up to allow vehicles that are driving at or too close to an intersection to pass safely through an intersection. Therefore, amber lights at traffic lights must be on long enough to prevent drivers approaching intersections from being caught in a dilemma. So the setting of amber light has a lot of restrictions, and it is important to optimize the road condition.

\section{Function Model of Amber Light Duration}

There are many relevant factors to be taken into account when establishing the time model of yellow light. In this paper, we consider the factors in the problem and consult the relevant data ${ }^{[1]}$, then conclude that the decisive factors in the calculation of the amber light duration are the speed of the vehicle, the width of the intersection, the length of the vehicle, driver's reaction time and braking distance.

First of all, according to the actual situation, we simulate the situation of the intersection, using 
Visio software to draw the image of the intersection.

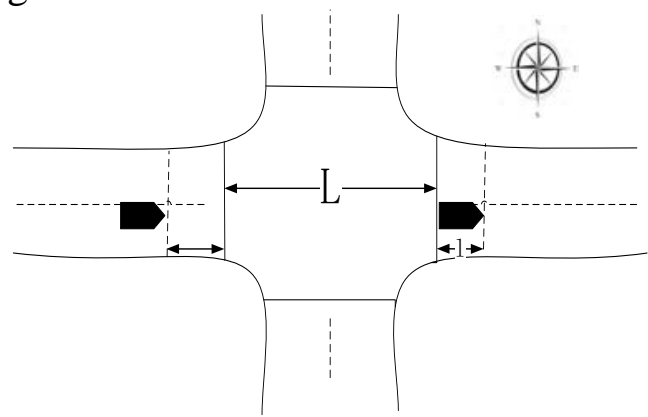

Figure 1 Crossroads

According to Figure 1, the situation at the crossroad can be clearly seen, in which the length of the car is $l$ and the width of the intersection is $D$. For this model, we have a more intuitive understanding by combining with reality.

Then, according to the known conditions, a mathematical model of the length of the amber light is established, which is related to the above factors (or part of the factors), and the length of the amber light is expressed as a function of the above factors (or parts of the factors). The model of the minimum distance vehicles can stop before the stop line is shown Equation (1) ${ }^{[2]}$.

$$
L=v_{0} t_{1}+\frac{v_{0}^{2}}{2 \mu g} \text {. }
$$

In Equation (1), $v_{0}$ represents the initial speed of a vehicle near the parking line, its unit is $\mathrm{m} / \mathrm{s}$. $t_{1}$ is reaction time, its unit is $\mathrm{s} . \mu$ is , $L$ represents braking distance, its unit is $\mathrm{m}$.

Amber light duration is shown in Equation (2).

$$
t=\frac{L+D+l}{v_{0}} .
$$

According to Equation (1) and (2), the function calculation model of amber light duration is finally got, shown in Equation (3).

$$
t=t_{1}+\frac{v_{0}}{2 \mu g}+\frac{D}{v_{0}}+\frac{L}{v_{0}} .
$$

In Equation (3), the amber light duration is $t$, the unit is $\mathrm{s}$, the width of the crossing is $D$, the unit is $m, l$ is the length of the vehicle, and the unit is $m$.

\section{Vehicle Speed and Amber Light Duration Model}

On the basis of the above problems, we add the known conditions. Assuming that the reaction time is $1 \mathrm{~s}$, the average length of the vehicle is $4.5 \mathrm{~m}$, the width of the intersection is $15 \mathrm{~m}$, and the friction coefficient is 0.2 . Then, we analyze the relationship between the speed of the vehicle and the length of the amber light.

First of all, we replace the $t_{1}=1 \mathrm{~s}, \quad l=4.5 \mathrm{~m}, \quad D=15 \mathrm{~m}, \quad u=0.2, \quad g=9.8 \mathrm{~m} / \mathrm{s}^{2}$ with the original amber light time function model. Then, the function expression we get is shown in Equation (4).

$$
t=2+\frac{v_{0}}{1.96}+\frac{15}{v_{0}} .
$$

So we got a model of the relationship between the length of the amber light and the speed of the 
car. According to this model, MATLAB is used to draw the relationship between speed and time, shown in Figure 2.

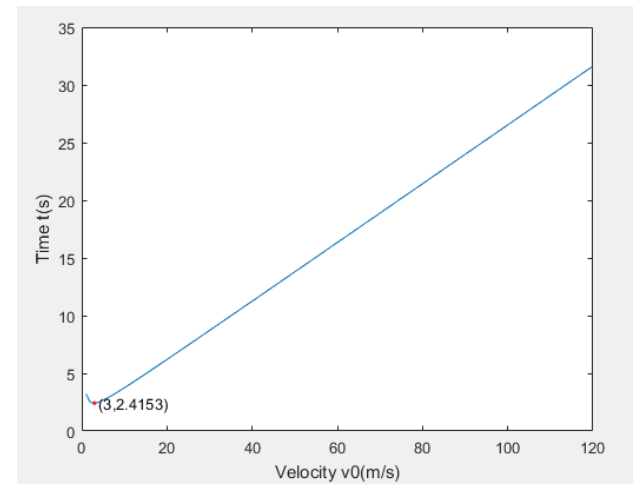

Figure 2 Relationship between Speed and Amber Light Duration 1

From Figure 2, it can be seen that amber light duration decreases with the increase of speed when $0 \leq v \leq 3$, and increases with the increase of speed when $v>10$. So we conclude the two are positively correlated.

Here, we think more deeply about this result. In actual life, the amber light duration should be increased with increasing speed, and there is no negative correlation in theory. After we find this, we deduce that the lowest speed of the car is $10 \mathrm{~km} / \mathrm{h}$ when the automobile runs normally through common sense, and according to the actual life and observation, the speed range of the car passing through the traffic light is about $10 \mathrm{~km} / \mathrm{h} \sim 30 \mathrm{~km} / \mathrm{h}$.

\section{Discussion on Amber Light Duration}

\subsection{Calculation I of Amber Light Duration}

We assume that the speed of the vehicle is $20 \mathrm{~km} / \mathrm{h}, 30 \mathrm{~km} / \mathrm{h}, 40 \mathrm{~km} / \mathrm{h}$ and $50 \mathrm{~km} / \mathrm{h}$, respectively. Friction coefficient $\mu$ is not a definite value because of friction coefficient. Many factors, such as weather, tire quality, will affect $\mu$. Assuming that the friction coefficient is increased from 0.2 to 0.4 , we use the MATLAB to obtain the relationship between the driving speed of the vehicle and the time length of the amber light, shown in Figure 3.

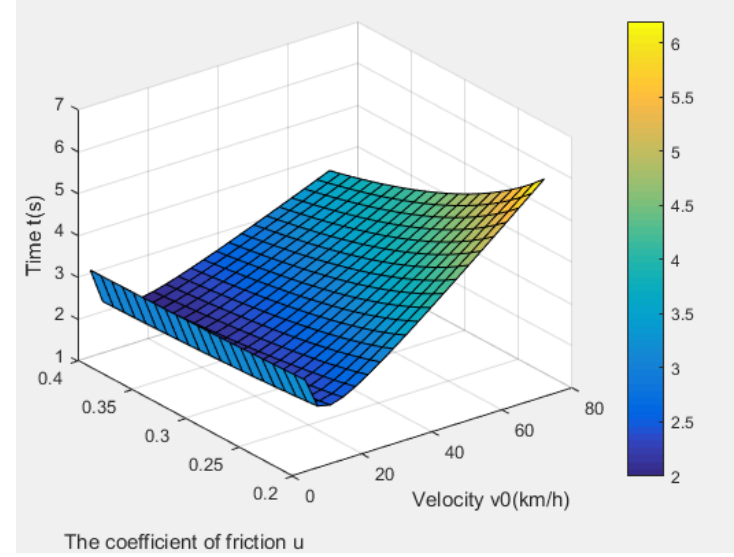

Figure 3 Relationship Between $\mu$, Speed and Amber Light Duration

In Figure 3, the 3D coordinates are vehicle speed $v_{0}$, friction coefficient $\mu$ and amber light duration $t$. The depth of color in the picture represents the length of the amber light. The three 
dimensional images show the general trend of the three: amber light decreases first and then increases with the increase of velocity. It decreases with the increase of friction coefficient. There is a general negative correlation.

Since the relationship between the friction coefficient and the length of the yellow light cannot be seen visually when the vehicle speed is small in the above image, we selected some representative points and obtained Table 1.

TABLE I. Amber Light Duration

\begin{tabular}{ccccc}
\hline$\mu$ & & & & \\
$v$ & 20 & 30 & 40 & 50 \\
\hline 0.2 & & & & \\
0.25 & 2.7682 & 3.3599 & 4.0100 & 4.6835 \\
0.3 & 2.4848 & 2.9347 & 3.4431 & 3.9749 \\
0.35 & 2.2958 & 2.6512 & 3.0651 & 3.5025 \\
0.4 & 2.0596 & 2.4488 & 2.7952 & 3.1650 \\
\hline
\end{tabular}

Compared with Figure 4, Table 2 shows more clearly the relationship between $\mu$ and amber light duration. With the increase of $\mu$, the duration decreases gradually.

\subsection{Calculation II of Amber Light Duration}

In the course of the study of the above model, some factors are calculated according to the given values, such as the width of the crossroads, which is not a fixed value. In addition to objective conditions some subjective conditions such as different traffic conditions and drivers' habits will have a greater impact on the calculation of amber light duration. After these factors are taken into account, the amber light duration is further solved and a final average value is calculated.

Here, assume that the speed of the crossing is between $20 \mathrm{~km} / \mathrm{h}$ and $50 \mathrm{~km} / \mathrm{h}$, with uniform distribution. And according to the existing research results, we know that: The perceptual time of $85 \%$ drivers is between ls and 1.8s, and for some older drivers, the reaction time is not less than 2.5s. According to the American Association of Highway and Transportation Workers, we take the reaction time as $2.5 \mathrm{~s}$. Therefore, in this question, we set the reaction time to $2.5 \mathrm{~s}$, the length of the vehicle to $4.5 \mathrm{~m}$, the width of crossroads to $25 \mathrm{~m}$ in general, and the dynamic friction factor of dry asphalt and concrete pavement to 0.7. The average amber light duration is shown in Equation (5).

$$
\bar{t}=\frac{\sum_{k=1}^{n} t_{k}}{n}=\frac{\sum_{k=1}^{n} t_{1}+\frac{v_{0 k}}{2 \mu g}+\frac{D+l}{v_{0 k}}}{n}, 1 \leq k \leq n .
$$

The above data are substituted into equation and solved by MATLAB, and the average amber light duration is $3.4231 \mathrm{~s}$.

\section{Acknowledgment}

Corresponding author Meng Zhaoping. E-mail address: Mengzhp@yeah.net. This work supported by the "12th Five-Year" Educational Science Plan of Shandong Province Grant No: ZBS15006. 


\section{References}

[1] Jiang Xin, Fan Lei and Tian Rui, “Study on the signal Model of Amber Light at Urban Signal Intersection," Inner Mongolia Highway and Transportation, vol. 2, Apr. 2010, pp. 54-56.

[2] Geng Aixia, Chen Qingmei and Xing Hong, "Study on the Problem of Automobile through Amber Light and its Teaching Apocalypse-A Perspective Based on the Representation of Original Physical Problems,” Physics teacher, vol. 8, Aug. 2013, pp. 24-25.

[3] Li Jianyang, Li Hao and Zhang Peng, "Sensitivity Analysis of the Factors Affecting the Peak Acceleration of Cargo Platform Landing,” Proceedings of the China Journal of Engineering Machinery, vol. 10, Nov. 2012, pp. $492-494$. 